\title{
Defining Spirituality at the End of Life
}

\section{Dear Editor:}

Vachon et al. ${ }^{1}$ performed a conceptual analysis of spirituality at the end of life by reviewing the empirical literature for definitional elements of the concept. Eleven themes were identified, including, for example, "meaning and purpose" and "developmental nature." Based on this work, a definition of spirituality at the end of life was provided as "a developmental and conscious process, characterized by two movements of transcendence; either deep within the self or beyond the self."

Spirituality is an ill-defined concept leading to similar problems in research and practice as with a concept such as quality of life, and promoting consensus on an inclusive definition may help to advance research and practice. We therefore appreciate the contribution of a literature review and comprehensive thematic analysis. We would like to add some suggestions and comments to their approach and resulting themes, and to the provided definition.

The themes do not seem to be mutually exclusive because the themes "self-transcendence" and "faith and beliefs" both include "faith in self." Moreover, the themes do not represent a consistent class of themes because they differ by nature. For example, "developmental nature" is an overarching theme that may refer to spiritual growth due to development in finding "meaning" as well as to a change in "appreciation of life." Similarly, "conscious nature" is assumed to be a characteristic of other themes, which means that it transcends other themes. Listing themes with different levels of abstraction may be inherent to the approach followed whereby themes are derived from categorized definitional elements. However, fitted in some conceptual and possibly hierarchical model may be more informative.

Furthermore, we see two basic problems with the definition of spirituality provided. The first is that including transcendence in the definition will not resolve the problem cited by the authors, namely that spirituality is a very abstract term to palliative care providers, and is therefore unlikely to guide clinical practice. While the definition alone may not be helpful, the more elaborate description in terms of the themes probably is. We learn from this that a clear single definition that captures all of the complexities may just not be feasible. The second definitional issue we would like to comment on is that the authors' emphasis on the conscious experience is problematic for the assessment of spirituality in many patients at the end of life. The authors explain that they "insist on the fact that spirituality should be described as a conscious experience [...] even if not highlighted in many articles," and that ". . . the capacity to reflect upon values and priorities are what makes an experience spiritual." The emphasis on the capacity to perform highly intellectual activities excludes many patients at the end of life from having spiritual experiences by this definition, excluding for example, many nursing homes patients and patients with mild or more severe cognitive problems due to end-stage disease. We are not sure that a spiritual experience can only be acknowledged as such when experienced fully conscious, and, perhaps more importantly for practice, we doubt if this emphasis will help improve spiritual care at the end of life.

We suggest an alternative approach to arrive at a definition, or better, a model representative of the empirical literature, which is to review measurable aspects of spirituality that are included in instruments used to measure spirituality in end-of-life populations in practice. It should avoid other abstract terms and apply to patients at the end of life whose consciousness is frequently suboptimal, and be useful in practice.

\section{Reference}

1. Vachon M, Fillion L, Achille M: A conceptual analysis of spirituality at the end of life. J Palliat Med 2009;12:53-59.

Address correspondence to: Jenny T. van der Steen, Ph.D. ${ }^{1,2^{*}}$ Marie-José H.E. Gijsberts, M.D. ${ }^{1,2}$

Michael A. Echteld, Ph.D. ${ }^{2}$

Martien T. Muller, Ph.D. ${ }^{1,2}$

Miel W. Ribbe, M.D., Ph.D. ${ }^{1}$

Luc Deliens, Ph.D. ${ }^{2}$

${ }^{1}$ Department of Nursing Home Medicine

${ }^{2}$ Department of Public and Occupational Health

VU University Medical Center

EMGO Institute for Health and Care Research

Van der Boechorststraat 7

Amsterdam 1081 BT

The Netherlands

E-mail: j.vandersteen@vumc.nl

${ }^{*}$ Corresponding author 


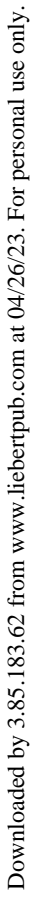

